\title{
NEW METHOD TO OBTAIN SMALL PARAMETER POWER SERIES EXPANSIONS OF MATHIEU RADIAL AND ANGULAR FUNCTIONS
}

\author{
T. M. LARSEN, D. ERRICOLO, AND P. L. E. USLENGHI
}

\begin{abstract}
Small parameter power series expansions for both radial and angular Mathieu functions are derived. The expansions are valid for all integer orders and apply the Stratton-Morse-Chu normalization. Three new contributions are provided: (1) explicit power series expansions for the radial functions, which are not available in the literature; (2) improved convergence rate of the power series expansions of the radial functions, obtained by representing the radial functions as a series of products of Bessel functions; (3) simpler and more direct derivations for the power series expansion for both the angular and radial functions. A numerical validation is also given.
\end{abstract}

\section{INTRODUCTION}

Mathieu angular and radial functions depend on a parameter $c$. This article describes a new method to compute their power series expansions when $0 \leq c<1$. Three new contributions are provided for small parameter Mathieu functions: (1) explicit power series expansions for the radial functions, which are not available in the literature; (2) improved convergence rate of the power series expansions of the radial functions, obtained by representing the radial functions as a series of products of Bessel functions; and, (3) simpler and more direct derivations for the power series expansion for both the angular and radial functions. Using this new method, power series expansions valid for all integer orders are obtained, using the Stratton-Morse-Chu normalization.

This work is motivated by physical applications where small parameter power series expansions for both angular and radial functions are required to develop analytical derivations. Power series expansions for Mathieu functions already present in the literature are not useful for the following two reasons. First, published results only contain expansions for the angular functions, but not for the radial functions, such as [1], 26], and 22]. Second, the normalization used is not appropriate. In fact, various normalizations exist for Mathieu functions and the two most frequently used are the one of Goldstein-Ince [17, 21] and the one of Stratton-Morse-Chu 37, 36]. When it comes to small parameter power series expansions, most published results apply the Goldstein-Ince normalization, such as [1], 26], with the exception of [22].

Received by the editor July 26, 2007 and, in revised form, October 26, 2007.

2000 Mathematics Subject Classification. Primary 33E10.

This work was supported by the U.S. Department of Defense under MURI grant F49620-011-0436 and by a Fellowship from the Aileen S. Andrew Foundation. The authors would like to thank the reviewers for their useful comments.

(C)2008 American Mathematical Society Reverts to public domain 28 years from publication 
The change of normalization is by no means a trivial operation when one needs to develop analytical derivations. Therefore, this article provides expressions for both radial and angular functions using the Stratton-Morse-Chu normalization. The choice of the Stratton-Morse-Chu normalization is dictated by a vast collection of analytical results that already exist, such as [5, 30, 7], 12, 13, 31, 14, 38, [39, 6].

This article fills a gap in the literature on Mathieu functions by extending and improving the results given in 22]. Preliminary results of this research were given in $[23$.

This work is organized as follows. The notation for the Matheiu functions is described in Section 2. Then, because the method to obtain the power series expansions differs between even and odd functions, even functions are examined in Section 3, and odd parity functions are considered in Section 4. The relationship with prior results obtained in [22] is clarified in Section 5, which is followed by a numerical validation in Section [6 and the conclusions in Section 7 .

\section{Mathieu ANGUlar and RAdial FUnctions}

Mathieu's differential equation is given by [36, 37]:

$$
\frac{\partial^{2}}{\partial v^{2}} f_{2}+\left(b-\frac{c^{2}}{2} \cos 2 v\right) f_{2}=0
$$

where $b$ and $c$ are constants. When $c>0$, Mathieu's equation contains a periodic coefficient. In many physical applications only periodic solutions are of interest, and for a given $c$ there exist two countable sets of values of $b$ for which equation (2.1) admits periodic solutions. These values of $b$ are called characteristic values and, depending upon the set, the period of the solution is either $\pi$ or $2 \pi$. Because of their periodicity and their meaning in physical applications, these solutions are also called Mathieu angular functions $\operatorname{Se}_{m}(c, v), \mathrm{So}_{m}(c, v)$. Closely related to (2.1) is Mathieu's modified differential equation [36, 37]:

$$
\frac{\partial^{2}}{\partial u^{2}} f_{1}+\left(\frac{c^{2}}{2} \cosh 2 u-b\right) f_{1}=0
$$

whose solutions are called Mathieu modified functions or Mathieu radial functions, $\operatorname{Re}_{m}^{(\ell)}(c, u), \operatorname{Ro}_{m}^{(\ell)}(c, u),(\ell=1, \ldots, 4)$, as suggested by many physical applications.

Mathieu functions were first introduced by Émile Léonard Mathieu (1835-1890) in 1868 when he investigated the vibrational modes of a stretched elliptical membrane 25. These functions have been studied by many authors, including Whittaker and Watson [41, Stratton [37, Morse and Feshbach [28], Meixner and Schäfke 27, and McLachlan 26]. The computation of Mathieu functions is not a trivial problem and it has been addressed by many authors, including Blanch [4, Clemm 8, 9, Hodge 20, Frisch 16], Rengarajan and Lewis 29, Baker [3, Shirts 34, 35, IMSL [40, Zhang and Jin [43], Alhargan [2, Mathematica 42], Erricolo [15.

Mathieu functions are used in many applications in physics and engineering, which are usually related to the solution of the wave equation in elliptic cylinder coordinates [5], [18, 33], 7], 32, [38, 13, 12, 24, 14, 39].

Both the angular and radial functions are expressed as series expansions. For the same order and parity, the expansion coefficients that define the angular and radial functions are the same. The expansion coefficients are not uniquely defined 
and different choices are possible depending upon the normalization used. In this work, only the Stratton-Morse-Chu normalization is considered.

\section{ExPANSIONS OF THE EVEN FUNCTIONS}

The definitions for both the angular and radial functions are provided by G. Blanch in [36. The series representing the even solutions of (2.1) and (2.2) are given by, respectively,

$$
\operatorname{Re}_{m}^{(1)}(c, u)=\sqrt{\frac{\pi}{2}} \sum_{k=0}^{\infty} j^{m-(2 k+p)} \frac{D_{2 k+p}^{m}}{D_{p}^{m}}\left[J_{k+p}\left(x_{1}\right) J_{k}\left(x_{2}\right)+p J_{k}\left(x_{1}\right) J_{k+p}\left(x_{2}\right)\right] .
$$

To obtain faster convergence for the even radial Mathieu functions, (3.2) contains a series of products of Bessel functions [36. The even radial Mathieu functions of the second kind and order $m$ are obtained by substituting $J_{k+p}\left(x_{1}\right)$ and $J_{k}\left(x_{1}\right)$ in (3.2) with their respective Bessel functions of the second kind, $N_{k+p}\left(x_{1}\right)$ and $N_{k}\left(x_{1}\right)$ :

$$
\operatorname{Re}_{m}^{(2)}(c, u)=\sqrt{\frac{\pi}{2}} \sum_{k=0}^{\infty} j^{m-(2 k+p)} \frac{D_{2 k+p}^{m}}{D_{p}^{m}}\left[N_{k+p}\left(x_{1}\right) J_{k}\left(x_{2}\right)+p N_{k}\left(x_{1}\right) J_{k+p}\left(x_{2}\right)\right] .
$$

In equations (3.1)-(3.3), $p=0$ if $m$ is even and $p=1$ if $m$ is odd. The arguments of the Bessel functions in (3.2) and (3.3) are:

$$
x_{1}=\frac{c}{2} e^{u}, \quad x_{2}=\frac{c}{2} e^{-u} .
$$

The even radial Mathieu functions of the third and fourth kind are defined by a linear combination of (3.2) and (3.3) 37, 36:

$$
\begin{aligned}
& \operatorname{Re}_{m}^{(3)}(c, u)=\operatorname{Re}_{m}^{(1)}(c, u)+j \operatorname{Re}_{m}^{(2)}(c, u), \\
& \operatorname{Re}_{m}^{(4)}(c, u)=\operatorname{Re}_{m}^{(1)}(c, u)-j \operatorname{Re}_{m}^{(2)}(c, u) .
\end{aligned}
$$

The expansion coefficients in (3.1), (3.2) and (3.3) and the even characteristic values are determined by the following second order recurrence relation [22]:

$$
\left(-n^{2}+b_{m}^{(e)}\right) D_{n}^{m}-\frac{c^{2}}{4}\left[D_{n+2}^{m}+\frac{2}{\varepsilon_{n-2}} D_{n-2}^{m}+\left(2-\varepsilon_{n-1}\right) D_{n}^{m}\right]=0, \quad m, n \geq 0 .
$$

Here $\varepsilon_{i}$ is the Neumann factor where $\varepsilon_{0}=1, \varepsilon_{i}=2$ for $i \neq 0$ and $n=2 k+p$. The expansion coefficients $D_{-n}^{m}=0$ for $n>0$. The $D_{n}^{m}(c)$ are expanded in a power series valid for small values of $c$ and have the form

$$
D_{n}^{m}(c)=\sum_{r=0}^{\infty} \alpha_{m, n}^{2 r} c^{2 r}
$$

where $\alpha_{m,-n}^{2 r}=0$ for $n>0$. When $c \rightarrow 0$, (2.1) becomes the equation of the harmonic oscillator with even solutions:

$$
\mathrm{Se}_{m}(0, v)=A \cos m v .
$$

Substituting (3.8) into (3.1) and setting $c=0$, we see that the expansion coefficients $\alpha_{m, m}^{0}=A$ and $\alpha_{m, n}^{0}=0(n \neq m)$ so that the only nonzero coefficient in (3.1) 
is $D_{m}^{m}(0)=A$. The constant $A$ will later be determined by the normalization introduced by Stratton and Chu.

The power series expansion for the even characteristic values have the form [22]

$$
b_{m}^{(e)}(c)=\sum_{j=0}^{\infty} l_{2 j}^{(e)} c^{2 j},
$$

where $l_{2 j}^{(e)}$ are even expansion coefficients and $l_{0}^{(e)}=m^{2}$, since $b_{m}^{(e)}(0)=m^{2}$ when $c \rightarrow 0$ in 2.1). The normalization, used by Stratton and Chu, is chosen so that $S e_{m}(c, 0)=1$ [37], or similarly,

$$
\sum_{k=0}^{\infty} D_{2 k+p}^{m}(c)=1 .
$$

Substitution of (3.8) into (3.11) leads to:

$$
\sum_{r=0}^{\infty}\left(\sum_{k=0}^{\infty} \alpha_{m, 2 k+p}^{2 r}\right) c^{2 r}=1
$$

From (3.12) and the comments above, we see that the normalization is satisfied by the following relations:

$$
\begin{aligned}
\alpha_{m, m}^{0} & =1 \quad(r=0, \quad A=1), \\
\sum_{k=0}^{\infty} \alpha_{m, 2 k+p}^{2 r} & =0 \quad(r \geq 1) .
\end{aligned}
$$

The expansion coefficients are obtained by substituting (3.8) and (3.10) into (3.7) and equating coefficients of like powers of $c$. The normalization is then satisfied by use of (3.13) and (3.14). Substituting (3.8) and (3.10) into (3.7) leads to:

$$
\begin{aligned}
\sum_{r=0}^{\infty} & \left(m^{2}-n^{2}\right) \alpha_{m, n}^{2 r} c^{2 r} \\
= & \frac{1}{4} \sum_{s=0}^{\infty}\left[\alpha_{m, n+2}^{2 s}+\frac{2}{\varepsilon_{n-2}} \alpha_{m, n-2}^{2 s}+\left(2-\varepsilon_{n-1}\right) \alpha_{m, n}^{2 s}\right] c^{2 s+2} \\
& -\sum_{p=0}^{\infty} \sum_{j=1}^{\infty} l_{2 j}^{(e)} \alpha_{m, n}^{2 p} c^{2(p+j)}
\end{aligned}
$$

Equating coefficients of $c^{0}(r=0)$ in (3.15) leads to

$$
\left(m^{2}-n^{2}\right) \alpha_{m, n}^{0}=0 .
$$

Equation (3.16) restates that $\alpha_{m, m}^{0}(n=m)$ is the only nonzero coefficient when $r=0$. Since (3.16) is satisfied for all values of $\alpha_{m, m}^{0}$, it must be determined by the normalization given by (3.13) $\left(\alpha_{m, m}^{0}=1\right)$. Therefore, the first nonzero expansion coefficients correspond to $n=m$ and are given by:

$$
\begin{aligned}
l_{0}^{(e)} & =m^{2}, \\
\alpha_{m, m}^{0} & =1 .
\end{aligned}
$$


Equating coefficients of $c^{2}(r=1)$ in (3.15) leads to:

$$
\left(m^{2}-n^{2}\right) \alpha_{m, n}^{2}=\frac{1}{4}\left\{\alpha_{m, n+2}^{0}+\frac{2}{\varepsilon_{n-2}} \alpha_{m, n-2}^{0}+\left[\left(2-\varepsilon_{n-1}\right)-4 l_{2}^{(e)}\right] \alpha_{m, n}^{0}\right\}
$$

In this case, all expansion coefficients are equal to zero for all $m$ except those corresponding to $n=m-2, n=m$ and $n=m+2$. Substituting these values of $n$ into (3.19) leads to

$$
\begin{aligned}
l_{2}^{(e)} & =\frac{2-\varepsilon_{m-1}}{4}, \\
\alpha_{m, m}^{2} & \neq 0, \\
\alpha_{m, m-2}^{2} & = \begin{cases}\frac{1}{16(m-1)}, & m \geq 2, \\
0, & m<2,\end{cases} \\
\alpha_{m, m+2}^{2} & = \begin{cases}-\frac{1}{16(m+1)}, & m \geq 1, \\
-\frac{1}{8}, & m=0,\end{cases}
\end{aligned}
$$

respectively. In equation (3.21), the value of $\alpha_{m, m}^{2}$ is generally nonzero. The appropriate value is determined by the normalization given by (3.14). Substitution of (3.22) and (3.23) into (3.14) $(r=1)$ leads to:

$$
\alpha_{m, m}^{2}= \begin{cases}-\frac{1}{8\left(m^{2}-1\right)}, & m \geq 2, \\ \frac{1}{32}, & m=1, \\ \frac{1}{8}, & m=0 .\end{cases}
$$

Equating coefficients of $c^{4}(r=2)$ in (3.15) leads to:

$$
\begin{aligned}
& \left(m^{2}-n^{2}\right) \alpha_{m, n}^{4} \\
& =\frac{1}{4}\left\{\alpha_{m, n+2}^{2}+\frac{2}{\varepsilon_{n-2}} \alpha_{m, n-2}^{2}+\left[\left(2-\varepsilon_{n-1}\right)-4 l_{2}^{(e)}\right] \alpha_{m, n}^{2}-4 l_{4}^{(e)} \alpha_{m, n}^{0}\right\}
\end{aligned}
$$

Here we find a total of five nonzero expansion coefficients corresponding to $n=$ $m-4, n=m-2, n=m, n=m+2$ and $n=m+4$. Setting $n=m$ and substituting (3.20) into (3.25), yields:

$$
\begin{gathered}
l_{4}^{(e)}= \begin{cases}\frac{1}{32\left(m^{2}-1\right)}, & m=0 \text { or } m \geq 3, \\
\frac{5}{192}, & m=2, \\
-\frac{1}{128}, & m=1,\end{cases} \\
\alpha_{m, m}^{4} \neq 0 .
\end{gathered}
$$


Setting $n=m-4, n=m-2, n=m+2$ and $n=m+4$ in (3.25) leads to

$$
\begin{aligned}
& \alpha_{m, m-4}^{4}= \begin{cases}\frac{1}{512(m-2)(m-1)}, & m \geq 4, \\
0, & m<4,\end{cases} \\
& \alpha_{m, m-2}^{4}= \begin{cases}-\frac{1}{128\left(m^{2}-1\right)(m-1)}, & m=2 \text { or } m \geq 4, \\
\frac{1}{2048}, & m=3, \\
0, & m<2,\end{cases} \\
& \alpha_{m, m+2}^{4}= \begin{cases}\frac{1}{128\left(m^{2}-1\right)(m+1)}, & m \geq 2, \\
-\frac{1}{512}, & m=1, \\
-\frac{1}{64}, & m=0,\end{cases} \\
& \alpha_{m, m+4}^{4}= \begin{cases}\frac{1}{512(m+2)(m+1)}, & m \geq 1, \\
\frac{1}{512}, & m=0,\end{cases}
\end{aligned}
$$

respectively. In equation (3.27), the value of $\alpha_{m, m}^{4}$ is generally nonzero. The appropriate value is determined by the normalization given by (3.14). Substitution of (3.28), (3.29), (3.30) and (3.31) into (3.14) $(r=2)$ leads to:

$$
\alpha_{m, m}^{4}= \begin{cases}-\frac{m^{4}-3 m^{2}+14}{256\left(m^{2}-1\right)^{2}\left(m^{2}-4\right)}, & m \geq 4 \\ -\frac{17}{20480}, & m=3 \\ \frac{29}{18432}, & m=2 \\ \frac{5}{3072}, & m=1 \\ \frac{7}{512}, & m=0\end{cases}
$$

The remaining expansion coefficients, $\alpha_{m, n}^{2 r}(r \geq 3)$, are determined by equating coefficients of $c^{2 r}$ in (3.15), substituting previously determined l's and expansion coefficients, and determining $\alpha_{m, m}^{2 r}$ by the normalization requirement of (3.14).

The power series expansions of the even Mathieu functions (i.e. in terms of the $\alpha$ expansion coefficients) are obtained by substitution of (3.8) into (3.1) and (3.2), respectively

$$
\begin{aligned}
& \operatorname{Se}_{m}(c, v)=\sum_{r=0}^{\infty}\left\{\sum_{k=0}^{\infty} \alpha_{m, 2 k+p}^{2 r} \cos (2 k+p) v\right\} c^{2 r} \\
& \operatorname{Re}_{m}^{(1)}(c, u)=\sqrt{\frac{\pi}{2}}\left(D_{p}^{m}\right)^{-1} \\
& \times \sum_{r=0}^{\infty}\left\{\sum_{k=0}^{\infty} j^{m-(2 k+p)} \alpha_{m, 2 k+p}^{2 r}\left[J_{k+p}\left(x_{1}\right) J_{k}\left(x_{2}\right)+p J_{k}\left(x_{1}\right) J_{k+p}\left(x_{2}\right)\right]\right\} c^{2 r}
\end{aligned}
$$


Setting $2 k+p=m \pm 2 q$ and keeping in mind the expansion coefficients which are equal to zero for all $m$, equations (3.14), (3.33) and (3.34) become, respectively,

$$
\begin{aligned}
& \alpha_{m, m}^{2 r}=-\sum_{q=1}^{r}\left\{\alpha_{m, m-2 q}^{2 r}+\alpha_{m, m+2 q}^{2 r}\right\} \\
& \operatorname{Se}_{m}(c, v)=\sum_{r=0}^{\infty}\left[\alpha_{m, m}^{2 r} \cos m v+\sum_{q=1}^{r}\left\{\alpha_{m, m-2 q}^{2 r} \cos [(m-2 q) v]\right.\right. \\
&\left.\left.+\alpha_{m, m+2 q}^{2 r} \cos [(m+2 q) v]\right\}\right] c^{2 r}
\end{aligned}
$$

$$
\begin{aligned}
& \operatorname{Re}_{m}^{(1)}(c, u)=\sqrt{\frac{\pi}{2}} \\
& \times \frac{\sum_{r=0}^{\infty}\left[\alpha_{m, m}^{2 r} J e_{\nu}(c, u)+\sum_{q=1}^{r}(-1)^{q}\left\{\alpha_{m, m-2 q}^{2 r} J e_{\gamma}(c, u)+\alpha_{m, m+2 q}^{2 r} J e_{\lambda}(c, u)\right\}\right] c^{2 r}}{\sum_{r=0}^{\infty} \alpha_{m, p}^{2 r} c^{2 r}},
\end{aligned}
$$

where the following substitutions have been made in (3.37):

$$
\begin{array}{r}
J e_{x}(c, u)=J_{x^{+}}\left(x_{1}\right) J_{x^{-}}\left(x_{2}\right)+p J_{x^{-}}\left(x_{1}\right) J_{x^{+}}\left(x_{2}\right), \\
\nu^{ \pm}=\frac{m \pm p}{2}, \quad \gamma^{ \pm}=\frac{m-2 q \pm p}{2}, \quad \lambda^{ \pm}=\frac{m+2 q \pm p}{2} .
\end{array}
$$

The summations in equations (3.36) and (3.37) extended from $q=1$ to $q=r$ are equal to zero when $r=0$. In practice, one evaluates

$$
\begin{aligned}
& \operatorname{Re}_{m}^{(1), t}(c, u)=\sqrt{\frac{\pi}{2}} \\
& \times \frac{\sum_{r=0}^{t-1}\left[\alpha_{m, m}^{2 r} J e_{\nu}(c, u)+\sum_{q=1}^{r}(-1)^{q}\left\{\alpha_{m, m-2 q}^{2 r} J e_{\gamma}(c, u)+\alpha_{m, m+2 q}^{2 r} J e_{\lambda}(c, u)\right\}\right] c^{2 r}}{\sum_{r=0}^{t-1} \alpha_{m, p}^{2 r} c^{2 r}},
\end{aligned}
$$

where $t=\left(\frac{m}{2}+N\right)$ or $t=\left(\frac{m-1}{2}+N\right)$ for $m$ even or odd, respectively, and $N \geq 1$ to obtain a good approximation.

The even radial Mathieu functions of the second kind along with the derivatives of the even radial Mathieu functions of the first and second kind (with respect to $u$ ) are obtained by replacing $J e_{x}(c, u)$ in (3.37) with $N e_{x}(c, u), J e_{x}^{\prime}(c, u)$ and $N e_{x}^{\prime}(c, u)$, respectively, which are given by:

$$
\begin{aligned}
N e_{x}(c, u)= & N_{x^{+}}\left(x_{1}\right) J_{x^{-}}\left(x_{2}\right)+p N_{x^{-}}\left(x_{1}\right) J_{x^{+}}\left(x_{2}\right), \\
J e_{x}^{\prime}(c, u)=\frac{1}{2} & x_{1}\left\{J_{x^{-}}\left(x_{2}\right)\left[J_{x^{+}-1}\left(x_{1}\right)-J_{x^{+}+1}\left(x_{1}\right)\right]\right. \\
& \left.+p J_{x^{+}}\left(x_{2}\right)\left[J_{x^{-}-1}\left(x_{1}\right)-J_{x^{-}+1}\left(x_{1}\right)\right]\right\} \\
& -x_{2}\left\{J_{x^{+}}\left(x_{1}\right)\left[J_{x^{-}-1}\left(x_{2}\right)-J_{x^{-}+1}\left(x_{2}\right)\right]\right. \\
& \left.\left.+p J_{x^{-}}\left(x_{1}\right)\left[J_{x^{+}-1}\left(x_{2}\right)-J_{x^{+}+1}\left(x_{2}\right)\right]\right\}\right],
\end{aligned}
$$




$$
\begin{aligned}
N e_{x}^{\prime}(c, u)=\frac{1}{2} & {\left[x_{1}\left\{J_{x^{-}}\left(x_{2}\right)\left[N_{x^{+}-1}\left(x_{1}\right)-N_{x^{+}+1}\left(x_{1}\right)\right]\right\}\right.} \\
& +p J_{x^{+}}\left(x_{2}\right)\left[N_{x^{-}-1}\left(x_{1}\right)-N_{x^{-}+1}\left(x_{1}\right)\right] \\
& -x_{2}\left\{N_{x^{+}}\left(x_{1}\right)\left[J_{x^{-}-1}\left(x_{2}\right)-J_{x^{-}+1}\left(x_{2}\right)\right]\right. \\
& \left.+p N_{x^{-}}\left(x_{1}\right)\left[J_{x^{+}-1}\left(x_{2}\right)-J_{x^{+}+1}\left(x_{2}\right)\right]\right\}
\end{aligned}
$$

The power series expansions for the first three terms of the even Mathieu functions can finally be obtained by substitution of (3.18), (3.22)-(3.24), (3.28)-(3.32) and (3.38)-(3.43) into the final expressions given by (3.5), (3.6), (3.36) and (3.37).

\section{EXPANSIONS OF THE ODD FUNCTIONS}

The determination of the power series expansions for the odd Mathieu functions follows the same procedure as for the even Mathieu functions with minor changes throughout the derivations. The odd solutions of (2.1) are expressed by the sine series:

$$
\mathrm{So}_{m}(c, v)=\sum_{k=(1-p)}^{\infty} F_{2 k+p}^{m} \sin (2 k+p) v \quad(m=1,2,3, \ldots),
$$

and odd solutions of (2.2) are expressed by the product Bessel series

$$
\begin{aligned}
\operatorname{Ro}_{m}^{(1)}(c, u)= & \sqrt{\frac{\pi}{2}} \sum_{k=(1-p)}^{\infty} j^{m-(2 k+p)} \frac{F_{2 k+p}^{m}}{F_{2-p}^{m}}\left[J_{k+1}\left(x_{1}\right) J_{k-1+p}\left(x_{2}\right)\right. \\
& \left.-J_{k-1+p}\left(x_{1}\right) J_{k+1}\left(x_{2}\right)\right],
\end{aligned}
$$

where the products of Bessel functions are introduced to increase the convergence rate [36]. The odd radial Mathieu functions of the second kind are expressed as:

$$
\begin{aligned}
\operatorname{Ro}_{m}^{(2)}(c, u)= & \sqrt{\frac{\pi}{2}} \sum_{k=(1-p)}^{\infty} j^{m-(2 k+p)} \frac{F_{2 k+p}^{m}}{F_{2-p}^{m}}\left[N_{k+1}\left(x_{1}\right) J_{k-1+p}\left(x_{2}\right)\right. \\
& \left.-N_{k-1+p}\left(x_{1}\right) J_{k+1}\left(x_{2}\right)\right] .
\end{aligned}
$$

In equations (4.1)-(4.3), $p=0$ if $m$ is even and $p=1$ if $m$ is odd. The arguments of the Bessel functions in (4.2) and (4.3) are given by equation (3.4). The odd radial Mathieu functions of the third and fourth kind are defined by a linear combination of (4.2) and (4.3) [37, 36]:

$$
\begin{aligned}
& \operatorname{Ro}_{m}^{(3)}(c, u)=\operatorname{Ro}_{m}^{(1)}(c, u)+j \operatorname{Ro}_{m}^{(2)}(c, u) \\
& \operatorname{Ro}_{m}^{(4)}(c, u)=\operatorname{Ro}_{m}^{(1)}(c, u)-j \operatorname{Ro}_{m}^{(2)}(c, u) .
\end{aligned}
$$

The expansion coefficients in (4.1), (4.2) and (4.3) and the odd characteristic values are determined by the following second order recurrence relation [22]:

$$
\left(-n^{2}+b_{m}^{(o)}\right) F_{n}^{m}-\frac{c^{2}}{4}\left[F_{n+2}^{m}+F_{n-2}^{m}-\left(2-\varepsilon_{n-1}\right) F_{n}^{m}\right]=0, \quad m, n \geq 1 .
$$

Here $n=2 k+p$, the Neumann factor has the same meaning as it did for the even functions and the expansion coefficients $F_{-n}^{m}=0$ for $n \geq 0$. The $F_{n}^{m}(c)$ are expanded in a power series valid for small values of $c$ and have the form

$$
F_{n}^{m}(c)=\sum_{r=0}^{\infty} \beta_{m, n}^{2 r} c^{2 r}
$$


where $\beta_{m,-n}^{2 r}=0$ for $n \geq 0$. When $c \rightarrow 0$ in (2.1), the odd harmonic solutions are given by:

$$
\mathrm{So}_{m}(0, v)=B \sin m v .
$$

Substituting (4.7) into (4.1) and setting $c=0$, we see that the expansion coefficients $\beta_{m, m}^{0}=B$ and $\beta_{m, n}^{0}=0(n \neq m)$ so that the only nonzero coefficient in (4.1) is $F_{m}^{m}(0)=B$. The constant $B$ will later be determined by the normalization.

The power series expansions for the odd characteristic values have the following form [22]:

$$
b_{m}^{(o)}(c)=\sum_{j=0}^{\infty} l_{2 j}^{(o)} c^{2 j}
$$

where $l_{2 j}^{(o)}$ are odd expansion coefficients and $l_{0}^{(o)}=m^{2}$, since $b_{m}^{(o)}(0)=m^{2}$ when $c \rightarrow 0$ in (2.1).

The normalization, used by Stratton and Chu, is chosen so that 37:

$$
\left[\frac{d}{d v} \operatorname{So}_{m}(c, v)\right]_{v=0}=1 \text {, }
$$

or similarly:

$$
\sum_{k=0}^{\infty}(2 k+p) F_{2 k+p}^{m}(c)=1
$$

Substitution of (4.7) into (4.10) leads to

$$
\sum_{r=0}^{\infty}\left[\sum_{k=0}^{\infty}(2 k+p) \beta_{m, 2 k+p}^{2 r}\right] c^{2 r}=1
$$

From (4.11) and the comments above, the normalization is satisfied by the following:

$$
\begin{aligned}
& \beta_{m, m}^{0}=\frac{1}{m} \quad\left(r=0, \quad B=\frac{1}{m}\right), \\
& \sum_{k=0}^{\infty}(2 k+p) \beta_{m, 2 k+p}^{2 r}=0 \quad(r \geq 1) .
\end{aligned}
$$

The expansion coefficients are obtained by substituting (4.7) and (4.9) into (4.6) and equating coefficients of like powers of $c$. The normalization is then satisfied by use of (4.12) and (4.13). Substituting (4.7) and (4.9) into (4.6) leads to:

$$
\begin{aligned}
& \sum_{r=0}^{\infty}\left(m^{2}-n^{2}\right) \beta_{m, n}^{2 r} c^{2 r} \\
& =\frac{1}{4} \sum_{s=0}^{\infty}\left[\beta_{m, n+2}^{2 s}+\beta_{m, n-2}^{2 s}-\left(2-\varepsilon_{n-1}\right) \beta_{m, n}^{2 s}\right] c^{2 s+2}-\sum_{p=0}^{\infty} \sum_{j=1}^{\infty} l_{2 j}^{(o)} \beta_{m, n}^{2 p} c^{2(p+j)} .
\end{aligned}
$$

Equating coefficients of $c^{0}(r=0)$ in (4.14) leads to:

$$
\left(m^{2}-n^{2}\right) \beta_{m, n}^{0}=0 \text {. }
$$

Equation (4.15) restates that $\beta_{m, m}^{0}(n=m)$ is the only nonzero coefficient when $r=0$. Since (4.15) is satisfied for all values of $\beta_{m, m}^{0}$, it must be determined by the 
normalization given by (4.12) $\left(\beta_{m, m}^{0}=\frac{1}{m}\right)$. Therefore, the first nonzero expansion coefficients correspond to $n=m$ and are given by:

$$
\begin{aligned}
l_{0}^{(o)} & =m^{2}, \\
\beta_{m, m}^{0} & =\frac{1}{m} .
\end{aligned}
$$

Equating coefficients of $c^{2}(r=1)$ in (4.14) leads to:

$$
\begin{aligned}
& \left(m^{2}-n^{2}\right) \beta_{m, n}^{2} \\
& \quad=\frac{1}{4}\left\{\beta_{m, n+2}^{0}+\beta_{m, n-2}^{0}-\left[\left(2-\varepsilon_{n-1}\right)+4 l_{2}^{(o)}\right] \beta_{m, n}^{0}\right\} .
\end{aligned}
$$

In this case, all expansion coefficients are equal to zero for all $m$ except those corresponding to $n=m-2, n=m$ and $n=m+2$. Substituting these values of $n$ into (4.18) leads to:

$$
\begin{aligned}
l_{2}^{(o)} & =\frac{\varepsilon_{m-1}-2}{4}, \\
\beta_{m, m}^{2} & \neq 0, \\
\beta_{m, m-2}^{2} & = \begin{cases}\frac{1}{16 m(m-1)}, & m \geq 3, \\
0, & m<3,\end{cases} \\
\beta_{m, m+2}^{2} & =-\frac{1}{16 m(m+1)}, \quad m \geq 1,
\end{aligned}
$$

respectively. In equation (4.20), the value of $\beta_{m, m}^{2}$ is generally nonzero. The appropriate value is determined by the normalization given by (4.13). Substitution of (4.21) and (4.22) into (4.13) $(r=1)$ leads to:

$$
\beta_{m, m}^{2}= \begin{cases}\frac{1}{8 m\left(m^{2}-1\right)}, & m \geq 3 \\ \frac{1}{48}, & m=2, \\ \frac{3}{32}, & m=1\end{cases}
$$

Equating coefficients of $c^{4}(r=2)$ in (4.14) leads to:

$$
\begin{aligned}
& \left(m^{2}-n^{2}\right) \beta_{m, n}^{4} \\
& \quad=\frac{1}{4}\left\{\beta_{m, n+2}^{2}+\beta_{m, n-2}^{2}-\left[\left(2-\varepsilon_{n-1}\right)+4 l_{2}^{(o)}\right] \beta_{m, n}^{2}-4 l_{4}^{(o)} \beta_{m, n}^{0}\right\} .
\end{aligned}
$$

Here we find a total of five nonzero expansion coefficients corresponding to $n=$ $m-4, n=m-2, n=m, n=m+2$ and $n=m+4$. Setting $n=m$ and substituting (4.19) in (4.24), we find that:

$$
\begin{aligned}
l_{4}^{(o)} & = \begin{cases}\frac{1}{32\left(m^{2}-1\right)}, & m \geq 3, \\
-\frac{1}{192}, & m=2, \\
-\frac{1}{128}, & m=1 .\end{cases} \\
\beta_{m, m}^{4} \neq 0 . &
\end{aligned}
$$


Setting $n=m-4, n=m-2, n=m+2$ and $n=m+4$ in (4.24) leads to

$$
\begin{aligned}
& \beta_{m, m-4}^{4}= \begin{cases}\frac{1}{512 m(m-2)(m-1)}, & m \geq 5, \\
0, & m<5,\end{cases} \\
& \beta_{m, m-2}^{4}= \begin{cases}\frac{1}{128 m\left(m^{2}-1\right)(m-1)}, & m \geq 4, \\
-\frac{1}{6144}, & m=3, \\
0, & m<3,\end{cases} \\
& \beta_{m, m+2}^{4}= \begin{cases}-\frac{1}{128 m\left(m^{2}-1\right)(m+1)}, & m \geq 3, \\
-\frac{1}{2304}, & m=2, \\
-\frac{1}{512}, & m=1,\end{cases} \\
& \beta_{m, m+4}^{4}=\frac{1}{512 m(m+2)(m+1)}, \quad m \geq 1 \text {, }
\end{aligned}
$$

respectively. In equation (4.26), the value of $\beta_{m, m}^{4}$ is generally nonzero. The appropriate value of this constant is determined by the normalization given by (4.13). Substitution of (4.27), (4.28), (4.29) and (4.30) into (4.13) $(r=2)$ leads to:

$$
\beta_{m, m}^{4}= \begin{cases}-\frac{m^{4}-15 m^{2}+26}{256 m\left(m^{2}-1\right)^{2}\left(m^{2}-4\right)}, & m \geq 5 \\ -\frac{7}{460800}, & m=4, \\ \frac{7}{61440}, & m=3, \\ \frac{23}{36864}, & m=2, \\ \frac{13}{3072}, & m=1 .\end{cases}
$$

The remaining expansion coefficients, $\beta_{m, n}^{2 r}(r \geq 3)$, are determined by equating coefficients of $c^{2 r}$ in (4.14), substituting previously determined l's and expansion coefficients, and determining $\beta_{m, m}^{2 r}$ by the normalization requirement of (4.13). The power series expansions of the odd Mathieu functions (i.e. in terms of the $\beta$ expansion coefficients) are obtained by substitution of (4.7) into (4.1) and (4.2), respectively,

$$
\begin{aligned}
& \operatorname{So}_{m}(c, v)=\sum_{r=0}^{\infty}\left\{\sum_{k=(1-p)}^{\infty} \beta_{m, 2 k+p}^{2 r} \sin (2 k+p) v\right\} c^{2 r}, \\
& \operatorname{Ro}_{m}^{(1)}(c, u)=\sqrt{\frac{\pi}{2}}\left(F_{2-p}^{m}\right)^{-1} \\
& \times \sum_{r=0}^{\infty}\left\{\sum _ { k = ( 1 - p ) } ^ { \infty } j ^ { m - ( 2 k + p ) } \beta _ { m , 2 k + p } ^ { 2 r } \left[J_{k+1}\left(x_{1}\right) J_{k-1+p}\left(x_{2}\right)\right.\right. \\
& \left.\left.\quad-J_{k-1+p}\left(x_{1}\right) J_{k+1}\left(x_{2}\right)\right]\right\} c^{2 r} .
\end{aligned}
$$


Setting $2 k+p=m \pm 2 q$ and keeping in mind the expansion coefficients which are equal to zero for all $m$, equations (4.13), (4.32) and (4.33) become respectively,

$$
\beta_{m, m}^{2 r}=-\frac{1}{m} \sum_{q=1}^{r}\left\{(m-2 q) \beta_{m, m-2 q}^{2 r}+(m+2 q) \beta_{m, m+2 q}^{2 r}\right\} \quad(r \geq 1),
$$

$$
\begin{aligned}
\operatorname{So}_{m}(c, v)= & \sum_{r=0}^{\infty}\left[\beta_{m, m}^{2 r} \sin m v+\sum_{q=1}^{r}\left\{\beta_{m, m-2 q}^{2 r} \sin [(m-2 q) v]\right.\right. \\
& \left.\left.+\beta_{m, m+2 q}^{2 r} \sin [(m+2 q) v]\right\}\right] c^{2 r}
\end{aligned}
$$

$$
\begin{aligned}
& \operatorname{Ro}_{m}^{(1)}(c, u)=\sqrt{\frac{\pi}{2}} \\
& \times \frac{\sum_{r=0}^{\infty}\left[\beta_{m, m}^{2 r} J o_{\nu}(c, u)+\sum_{q=1}^{r}(-1)^{q}\left\{\beta_{m, m-2 q}^{2 r} J o_{\gamma}(c, u)+\beta_{m, m+2 q}^{2 r} J o_{\lambda}(c, u)\right\}\right] c^{2 r}}{\sum_{r=0}^{\infty} \beta_{m, 2-p}^{2 r} c^{2 r}},
\end{aligned}
$$

where the following substitutions have been made in (4.36):

$$
\begin{gathered}
J_{o_{x}}(c, u)=J_{x^{+}}\left(x_{1}\right) J_{x^{-}}\left(x_{2}\right)-J_{x^{-}}\left(x_{1}\right) J_{x^{+}}\left(x_{2}\right), \\
\nu^{ \pm}=\frac{m \mp p \pm 2}{2}, \quad \gamma^{ \pm}=\frac{m-2 q \mp p \pm 2}{2}, \quad \lambda^{ \pm}=\frac{m+2 q \mp p \pm 2}{2} .
\end{gathered}
$$

The summations in equations (4.35) and (4.36) extended from $q=1$ to $q=r$ are equal to zero when $r=0$. In practice, one evaluates

$$
\operatorname{Ro}_{m}^{(1), t}(c, u)=\sqrt{\frac{\pi}{2}}
$$

$\times \frac{\sum_{r=0}^{t-1}\left[\beta_{m, m}^{2 r} J o_{\nu}(c, u)+\sum_{q=1}^{r}(-1)^{q}\left\{\beta_{m, m-2 q}^{2 r} J o_{\gamma}(c, u)+\beta_{m, m+2 q}^{2 r} J o_{\lambda}(c, u)\right\}\right] c^{2 r}}{\sum_{r=0}^{t-1} \beta_{m, 2-p}^{2 r} c^{2 r}}$,

where $t=\left(\frac{m}{2}-1+N\right)$ or $t=\left(\frac{m-1}{2}+N\right)$ when $m$ is even or odd, respectively, and $N \geq 1$ to obtain a good approximation.

The odd radial Mathieu functions of the second kind along with the derivatives of the odd radial Mathieu functions of the first and second kind (with respect to $u$ ) are obtained by replacing $J o_{x}(c, u)$ in (4.36) with $N o_{x}(c, u), J o_{x}^{\prime}(c, u)$ and $N o_{x}^{\prime}(c, u)$, 
respectively, which are given by:

$$
\begin{aligned}
& N o_{x}(c, u)=N_{x^{+}}\left(x_{1}\right) J_{x^{-}}\left(x_{2}\right)-N_{x^{-}}\left(x_{1}\right) J_{x^{+}}\left(x_{2}\right), \\
& J o_{x}^{\prime}(c, u)=\frac{1}{2}\left[x _ { 1 } \left\{J_{x^{-}}\left(x_{2}\right)\left[J_{x^{+}-1}\left(x_{1}\right)-J_{x^{++1}}\left(x_{1}\right)\right]\right.\right. \\
& \left.-J_{x^{+}}\left(x_{2}\right)\left[J_{x^{-}-1}\left(x_{1}\right)-J_{x^{-+1}}\left(x_{1}\right)\right]\right\} \\
& -x_{2}\left\{J_{x^{+}}\left(x_{1}\right)\left[J_{x^{-}-1}\left(x_{2}\right)-J_{x^{-}+1}\left(x_{2}\right)\right]\right. \\
& \left.\left.-J_{x^{-}}\left(x_{1}\right)\left[J_{x^{+-1}}\left(x_{2}\right)-J_{x^{+}+1}\left(x_{2}\right)\right]\right\}\right] \text {, } \\
& N o_{x}^{\prime}(c, u)=\frac{1}{2}\left[x _ { 1 } \left\{J_{x^{-}}\left(x_{2}\right)\left[N_{x^{+}-1}\left(x_{1}\right)-N_{x^{+}+1}\left(x_{1}\right)\right]\right.\right. \\
& \left.-J_{x^{+}}\left(x_{2}\right)\left[N_{x^{-}-1}\left(x_{1}\right)-N_{x^{-}+1}\left(x_{1}\right)\right]\right\} \\
& -x_{2}\left\{N_{x^{+}}\left(x_{1}\right)\left[J_{x^{-}-1}\left(x_{2}\right)-J_{x^{-}+1}\left(x_{2}\right)\right]\right. \\
& \text { - } \left.\left.N_{x^{-}}\left(x_{1}\right)\left[J_{x^{+}-1}\left(x_{2}\right)-J_{x^{+}+1}\left(x_{2}\right)\right]\right\}\right] \text {. }
\end{aligned}
$$

The power series expansions for the first three terms of the odd Mathieu functions can finally be obtained by substitution of (4.17), (4.21)-(4.23), (4.27)-(4.31) and (4.37)-(4.42) into the final expressions given by (4.4), (4.5), (4.35) and (4.36).

\section{Equivalence With the method of Kokkorakis and Roumeliotis}

The same results for the determination of the expansion coefficients for the power series expansions of the Mathieu functions can also be obtained using an alternate method introduced by Kokkorakis and Roumeliotis in 22. The results given in 22 are specifically derived for the angular functions, although they may also be used to determine the radial functions. Since the new derivations are shorter and simpler to understand, the advantages of the new method will be shown in the following, but only for the even angular functions since the derivations for the remaining functions are similar.

Both methods begin with the same definitions for the power series expansions of the even angular functions, given by (3.1), where the expansion coefficients are determined by the second order recurrence relation given by (3.7). Also, the power series expansion for $b_{m}^{(e)}(c)$ is the same in both methods and given by (3.10). The primary difference in 22] is the choice of the power series expression for the $D_{n}^{m}(c)$ given by

$$
D_{m \pm 2 q}^{m}(c)=\left[\sum_{k=0}^{\infty} \alpha_{2 q, 2 k}^{ \pm} c^{2 q+2 k}\right] D_{m}^{m}(c) \quad(q=0,1,2, \ldots),
$$

where $n=m \pm 2 q$. The $D_{m}^{m}(c)$ are also expanded in a power series

$$
D_{m}^{m}(c)=\sum_{l=0}^{\infty} g_{2 l} c^{2 l}
$$

where the normalization is satisfied by setting $g_{0}=1$, and

$$
g_{2 l}=-\sum_{q=1}^{l} \sum_{k=0}^{l-q} g_{2 k}\left[\alpha_{2 q, 2(l-q-k)}^{+}+\alpha_{2 q, 2(l-q-k)}^{-}\right] \quad(l=1,2, \ldots) .
$$

Comparing the expressions for $D_{n}^{m}(c)$ in both methods, we see that (3.8) is an 
infinite series where as (5.1) contains a product of two infinite series. This choice for the $D_{n}^{m}(c)$ leads to very lengthy algebraic calculations for the determination of the expansion coefficients that can be avoided by alternately using (3.8).

In this method, the expansion coefficients are determined by substituting (3.10) and (5.1) into (3.7) and equating coefficients of like powers of $c$ to zero. Once these substitutions are made, the $l_{2 j}^{(e)}$ are determined in a straightforward manner by setting $n=m$. On the other hand, the $\alpha_{2 q, 2 k}^{ \pm}$are determined by setting $n=m \pm 2 q$, which leads to the following recurrence relations:

$$
\begin{aligned}
& \alpha_{2 q, 0}^{ \pm}=f^{ \pm}(2 q-2) \alpha_{2 q-2,0}^{ \pm}, \quad q \geq 1, \quad k=0, \\
& \alpha_{2 q, 2}^{ \pm}=f^{ \pm}(2 q-2) \alpha_{2 q-2,2}^{ \pm}+g^{ \pm}(2 q) \alpha_{2 q, 0}^{ \pm}, \quad q \geq 1, \quad k=1, \\
& \alpha_{2 q, 2 k}^{ \pm}=f^{ \pm}(2 q-2) \alpha_{2 q-2,2 k}^{ \pm}+g^{ \pm}(2 q) \alpha_{2 q, 2 k-2}^{ \pm} \\
& \quad+t^{ \pm}(2 q) \sum_{j=2}^{k} l_{2 j}^{(e)} \alpha_{2 q, 2 k-2 j}^{ \pm}+p^{ \pm}(2 q+2) \alpha_{2 q+2,2 k-4}^{ \pm}, \quad q \geq 1, k \geq 2,
\end{aligned}
$$

where the following substitutions have been made:

$$
\begin{aligned}
t^{ \pm}(2 q) & = \pm \frac{1}{4 q(m \pm q)}, \\
f^{+}(2 q-2) & =-\frac{1}{2 \varepsilon_{m+2 q-2}} t^{+}(2 q), \quad f^{-}(2 q-2)=-\frac{1}{4} t^{-}(2 q), \\
g^{+}(2 q) & =\frac{2-\varepsilon_{m-1}}{4} t^{+}(2 q), \quad g^{-}(2 q)=-\frac{2-\varepsilon_{m-2 q-1}}{4} t^{-}(2 q), \\
p^{+}(2 q+2) & =-\frac{1}{4} t^{+}(2 q), \quad p^{-}(2 q+2)=-\frac{1}{2 \varepsilon_{m-2 q-2}} t^{-}(2 q) .
\end{aligned}
$$

The derivations for the expansion coefficients determined by (5.4)-(5.6) are straight forward when $k$ is small but become much more tedious as $k$ increases. Also, the derivations which lead to equations (5.3)-(5.10) are very tedious in itself. The final expression for the power series expansion of the even angular Mathieu functions is then given by:

$$
\begin{aligned}
& \operatorname{Se}_{m}(c, v)=\sum_{l=0}^{\infty} g_{2 l} c^{2 l} \\
& \times\left\{\cos m v+\sum_{q=1}^{\infty} \sum_{k=0}^{\infty}\left\{\alpha_{2 q, 2 k}^{+} \cos [(m+2 q) v]+\alpha_{2 q, 2 k}^{-} \cos [(m-2 q) v]\right\} c^{2(q+k)}\right\} .
\end{aligned}
$$

The first three terms are obtained by expanding (5.11) and setting the upper limits of the summations to the minimum values which lead to powers of $c^{0}, c^{2}$ and $c^{4}$. Expanding (5.11) and grouping together terms of like powers of $c$ leads to: 


$$
\begin{aligned}
& \mathrm{Se}_{m}(c, v) \approx \sum_{l=0}^{2} g_{2 l} c^{2 l} \\
& \quad \times\left\{\cos m v+\sum_{q=1}^{2} \sum_{k=0}^{1}\left\{\alpha_{2 q, 2 k}^{+} \cos [(m+2 q) v]+\alpha_{2 q, 2 k}^{-} \cos [(m-2 q) v]\right\} c^{2(q+k)}\right\},
\end{aligned}
$$

$$
\begin{aligned}
& \mathrm{Se}_{m}(c, v) \\
& =g_{0} \cos m v+\left\{g_{0} \alpha_{2,0}^{-} \cos [(m-2) v]+g_{2} \cos m v+g_{0} \alpha_{2,0}^{+} \cos [(m+2) v]\right\} c^{2} \\
& +\left\{g_{0} \alpha_{4,0}^{-} \cos [(m-4) v]+\left(g_{0} \alpha_{2,2}^{-}+g_{2} \alpha_{2,0}^{-}\right) \cos [(m-2) v]+g_{4} \cos m v\right. \\
& \left.+\left(g_{0} \alpha_{2,2}^{+}+g_{2} \alpha_{2,0}^{+}\right) \cos [(m+2) v]+g_{0} \alpha_{4,0}^{+} \cos [(m+4) v]\right\} c^{4}+O\left(c^{6}\right) .
\end{aligned}
$$

The meaning of the expansion coefficients used in both methods can be compared by setting the upper limit to $r=2$ in (3.36) and expanding the summation:

$$
\begin{aligned}
& \operatorname{Se}_{m}(c, v)=\alpha_{m, m}^{0} \cos m v \\
& +\left\{\alpha_{m, m-2}^{2} \cos [(m-2) v]+\alpha_{m, m}^{2} \cos m v+\alpha_{m, m+2}^{2} \cos [(m+2) v]\right\} c^{2} \\
& +\left\{\alpha_{m, m-4}^{4} \cos [(m-4) v]+\alpha_{m, m-2}^{4} \cos [(m-2) v]+\alpha_{m, m}^{4} \cos m v\right. \\
& \left.+\alpha_{m, m+2}^{4} \cos [(m+2) v]+\alpha_{m, m+4}^{4} \cos [(m+4) v]\right\} c^{4}+O\left(c^{6}\right) .
\end{aligned}
$$

Comparing (5.13) to (5.14) leads to the following relationships for the expansion coefficients:

$$
\begin{aligned}
\alpha_{m, m}^{2 r} & =g_{2 r}, \quad r \geq 0, \\
\alpha_{m, m \pm 2}^{2} & =g_{0} \alpha_{2,0}^{ \pm}, \\
\alpha_{m, m \pm 2}^{4} & =g_{0} \alpha_{2,2}^{ \pm}+g_{2} \alpha_{2,0}^{ \pm}, \\
\alpha_{m, m \pm 4}^{4} & =g_{0} \alpha_{4,0}^{ \pm},
\end{aligned}
$$

where the right side of equations (5.15)-(5.18) contain the expansion coefficients used in 22]. It is interesting to note the relationship between the $g_{2 l}$ in 22 ] and the $\alpha_{m, m}^{2 r}$. It is shown in (5.15) that these expansion coefficients are exactly equal. This should come as no surprise since they are determined by the normalization in both methods when $n=m$. Also from equations (5.16)-(15.18), we see that in general, the $\alpha_{m, n}^{2 r}$ when $n \neq m$ are related to the expansion coefficients obtained in [22] by the sum of products of the $g_{2 l}$ and $\alpha_{2 q, 2 k}^{ \pm}$. This is where it becomes apparent that the choice for the $D_{n}^{m}(c)$ given by (3.8) leads directly to the natural form of the power series expansion for $\operatorname{Se}_{m}(c, v)$.

\section{VALIDATION}

As an example of validation, we computed radial and angular Mathieu functions using the power series expansions given in this article and the numerical subroutines described in [15] that were independently validated in [10, 11. In all the following comparisons, the value $c=0.9$ is chosen for the small parameter because it provides a more challenging test than others with smaller values of $c$.

Even radial functions of the first kind are examined in Fig. 1 for $m=10$ and $0 \leq u \leq 5$. We evaluate $\operatorname{Re}_{m}^{(1), t}(c, u)$ of (3.40) and $\operatorname{Re}_{m}^{(1)}(c, u)$ with the numerical subroutines of [15]. Fig. 1(a) shows $\operatorname{Re}_{m}^{(1), t}(c, u)$ when $t=5$ (dotted line), $t=6$ 
(dashed line), and $\operatorname{Re}_{m}^{(1)}(c, u)$. Fig. $1(\mathrm{~b})$ shows $\operatorname{Re}_{m}^{(1), t}(c, u)$ with $t=7$ (dashed line) and $\operatorname{Re}_{m}^{(1)}(c, u)$. When 7 terms are considered, the small parameter expansion is not distinguishable from the reference solution obtained with independent numerical subroutines. To emphasize differences between the two evaluations, Fig. 3(a) shows the difference between $\operatorname{Re}_{m}^{(1), t}(c, u)$, with $t=7$, and $\operatorname{Re}_{m}^{(1)}(c, u)$.

Odd radial functions of the first kind are examined in Fig. 2 when $m=3$ and $0 \leq u \leq 5$. We evaluate $\operatorname{Ro}_{m}^{(1), t}(c, u)$ of (4.39) and $\operatorname{Ro}_{m}^{(1)}(c, u)$ with the numerical subroutines of [15]. Fig. $2(\mathrm{a}) \operatorname{shows} \operatorname{Ro}_{m}^{(1), t}(c, u)$ with $t=1$ (dotted line) and $t=2$ (dashed line) and $\operatorname{Ro}_{m}^{(1)}(c, u)$. Fig. 2(b) shows $\operatorname{Ro}_{m}^{(1), t}(c, u)$ with $t=3$ (dashed line) and $\operatorname{Ro}_{m}^{(1)}(c, u)$. When only 3 terms are computed, the curves shown in Fig. 2(b) overlap; however, to emphasize their differences Fig. 3(b) shows a plot of the difference between $\operatorname{Ro}_{m}^{(1), t}(c, u)$ and $\operatorname{Ro}_{m}^{(1)}(c, u)$.

These two comparisons emphasize the general behavior that the number of terms $t$ increases with the order $m$. This is obvious by the exam of Fig. 1(b) and the difference shown in Fig. 3(a) for $m=10$ versus Fig. 2(b) and the difference shown in Fig. 3(b) when $m=3$. Additionally, when $u=0, \operatorname{Re}_{m}^{(1), t}(c, u)=0$, from Fig. 1(b) and $d \operatorname{Ro}_{m}^{(1), t}(c, u) / d u=0$, as it may be inferred from Fig. 2(b) The last two features provide further confidence that our results are correct.

Angular functions are examined in Fig. 4 for $m=10$ and $0 \leq v \leq 2 \pi$. We indicate with the symbol $\mathrm{Se}_{m}^{t}(c, v)$ the evaluation of the first $t$ terms of equation (3.36) and with $\operatorname{Se}_{m}(c, v)$ the evaluation with the numerical subroutines of [15]. Fig. 4(a) shows $\operatorname{Se}_{m}^{t}(c, v)$ evaluated with $t=3$ (dashed line) and $\operatorname{Se}_{m}(c, v)$ (solid line). Since the two curves overlap, we need to examine the difference $\operatorname{Se}_{m}^{t}(c, v)-\operatorname{Se}_{m}(c, v)$ shown in Fig. 4(b) It is clear that only $t=3$ terms provide an excellent agreement with the reference solution. Additionally, Fig. 4(a) shows that $\operatorname{Se}_{m}(c, v)=1$ when $v=0$, which satisfies the requested Stratton-Morse-Chu normalization.

A validation for odd angular functions is not reported here because it leads to equivalent results and is omitted for brevity.

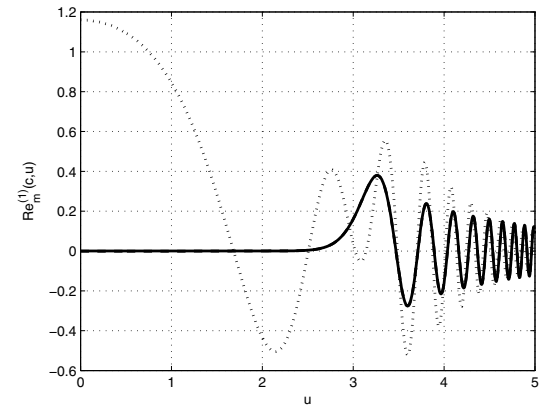

(a) $\operatorname{Re}_{m}^{(1), t}(c, u)$ evaluated with 5 terms (dotted line), 6 terms (dashed line) and $\operatorname{Re}_{m}^{(1)}(c, u)$ (solid line).

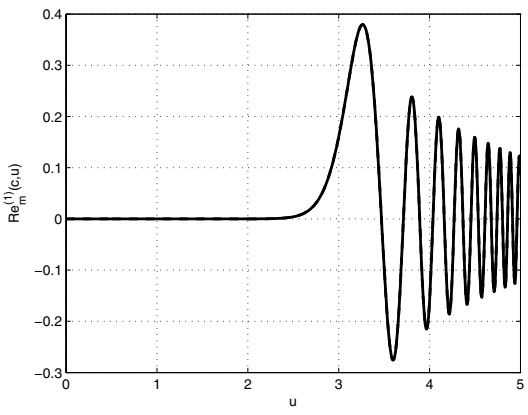

(b) $\operatorname{Re}_{m}^{(1), t}(c, u)$ evaluated with 7 terms (dashed line) and $\operatorname{Re}_{m}^{(1)}(c, u)$ (solid line).

Figure 1. Comparison between the evaluation of the small parameter expansion of the even radial functions of the first kind given in equation (3.37) and the numerical subroutines described in [15] for $m=10$. 


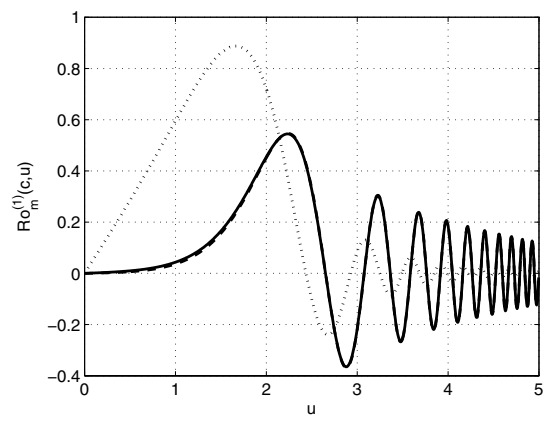

(a) $\operatorname{Ro}_{m}^{(1), t}(c, u)$ evaluated with 1 terms (dotted line), 2 terms(dashed line) and $\operatorname{Ro}_{m}^{(1)}(c, u)$ (solid line).

Figure 2. Comparison between the evaluation of the small parameter expansion of the odd radial functions of the first kind given in equation (4.36) and the numerical subroutines described in [15] for $m=3$.

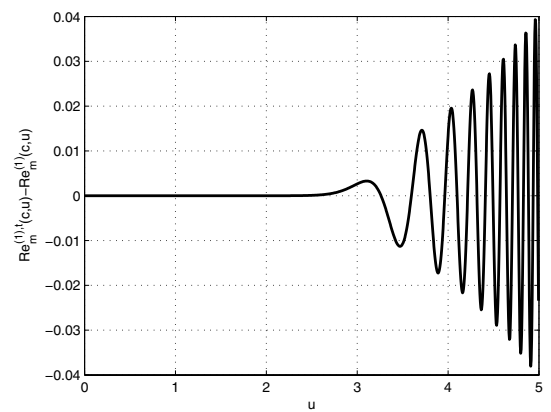

(a) Error in the evaluation of $\operatorname{Re}_{m}^{(1)}(c, u)$ using the small parameter approximation

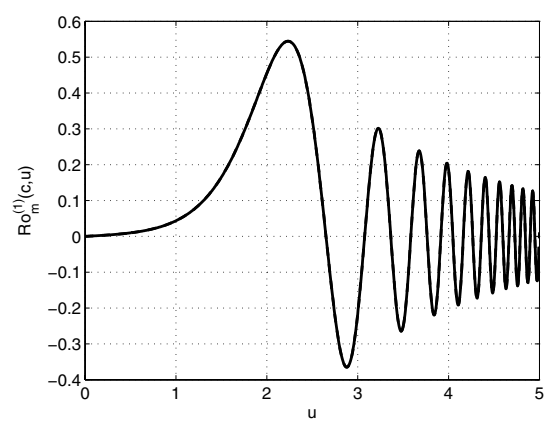

(b) $\operatorname{Ro}_{m}^{(1), t}(c, u)$ evaluated with 3 terms (dashed line) and $\operatorname{Ro}_{m}^{(1)}(c, u)$ (solid line).

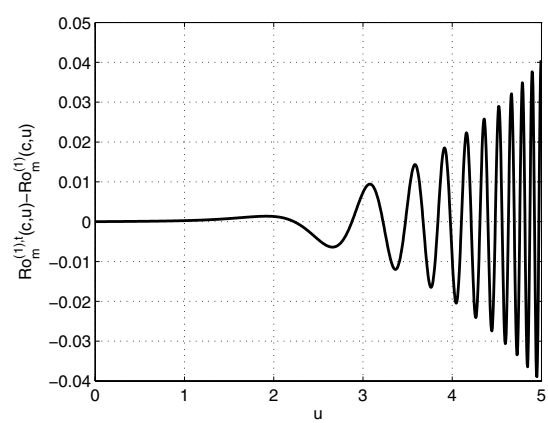

(b) Error in the evaluation of $\operatorname{Ro}_{m}^{(1)}(c, u)$ using the small parameter approximation

FIGURE 3. Even radial function of the first kind: comparison between small parameter approximation and numerical subroutines

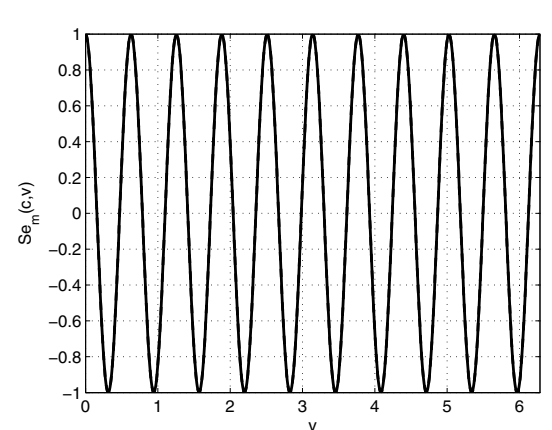

(a) $\operatorname{Se}_{m}^{t}(c, v)$ when $t=3$ (dashed line) and $\mathrm{Se}_{m}(c, v)$ (solid line)

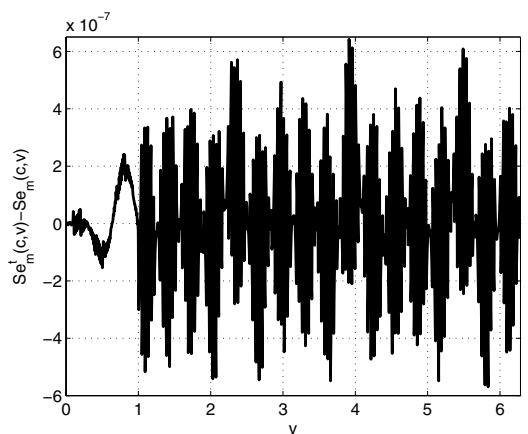

(b) Error in the evaluation of $\operatorname{Se}_{m}(c, v)$ using the small parameter expansion

FiguRE 4. Evaluation of the even angular function for $m=10$. 


\section{Conclusion}

This work provides explicit power series expansions for the Mathieu radial functions when the parameter $0 \leq c<1$ and using the Stratton-Morse-Chu normalization. To the best of the authors' knowledge, this is the first time this result is given in the literature.

Explicit power series expansions are also given for the Mathieu angular functions and the new results are in agreement with those previously obtained by Kokkorakis and Roumeliotis in [22]. The method used to obtain the power series expansions is an improvement over the one presented in [22] and it has three advantages:

(1) It is more straightforward and direct to apply.

(2) It leads to a linear complexity of the process to obtain the expansion coefficients.

(3) It leads to an easier software implementation.

Numerical validations show the good agreement of the new power series expansions with other numerical software to evaluate Mathieu functions.

Finally, as an example, the application that motivated this work is the extraction of the small parameter expansions of the results obtained by Uslenghi in [38] and Valentino and Erricolo in [39. In that context, the small parameter expansions correspond to low-frequency approximations, which could then be compared with quasi-static methods, such as those derived by Hansen and Yaghjian [19].

\section{REFERENCES}

[1] M. Abramovitz and I. A. Stegun, Handbook of mathematical functions, Dover Publications, Inc, New York, 1970.

[2] F. A. Alhargan, Algorithms for the computation of all Mathieu functions of integer order, ACM Trans. Math. Softw. 26 (2001), no. 3, 390-407.

[3] L. Baker, Mathematical function handbook, McGraw-Hill, 1992.

[4] G. Blanch, Numerical aspects of Mathieu eigenvalues, Rend. Circ. Mat. Paler., 2, vol. 15, 1966, pp. 51-97. MR0229377 (37:4951)

[5] J. J. Bowman, T. B. A. Senior, and P. L. E. Uslenghi, Electromagnetic and acoustic scattering by simple shapes, Hemisphere Publishing Corporation, New York, 1987. MR1111017 (92f:78014)

[6] S. M. Canta and D. Erricolo, Exact 2D Scattering Analysis of a slot backed by a semielliptical cavity and covered by a multilayer diaphragm, Radio Sci. (2007), submitted.

[7] S. Caorsi, M. Pastorino, and M. Raffetto, Electromagnetic scattering by a multilayer elliptic cylinder under transverse-magnetic illumination: series solution in terms of Mathieu functions, IEEE Trans. Antennas Propagat. 45 (1997), no. 6, 926-935.

[8] D. S. Clemm, Algorithm 352 Characteristic values and associated solutions of Mathieu's differential equation [S22], Commun. ACM 12 (1969), no. 7, 399-407.

[9] , Remark on algorithm 352 [S22] Characteristic values and associated solutions of Mathieu's differential equation, Commun. ACM 13 (1970), no. 12, 750.

[10] D. Erricolo, M. D. Lockard, C. M. Butler, and P. L. E. Uslenghi, Numerical analysis of penetration, radiation, and scattering for a $2 D$ slotted semielliptical channel filled with isorefractive material, PIER 53 (2005), 69-89.

[11] D. Erricolo, M. D. Lockard, C. M. Butler, and P. L. E. Uslenghi, Currents on conducting surfaces of a semielliptical-channel-backed slotted screen in an isorefractive environment, IEEE Trans. Antennas Propagat. 53 (2005), no. 7, 2350-2356.

[12] D. Erricolo and P. L. E. Uslenghi, Exact radiation and scattering for an elliptic metal cylinder at the interface between isorefractive half-spaces, IEEE Trans. Antennas Propagat. 52 (2004), no. 9, 2214-2225.

[13] — Penetration, radiation, and scattering for a cavity-backed gap in a corner, IEEE Trans. Antennas Propagat. 53 (2005), no. 8, 2738-2748. 
[14] D. Erricolo, P.L.E. Uslenghi, B. Elnour, and F. Mioc, Scattering by a blade on a metallic plane, Electromagnetics 26 (2005), no. 1, 57-71.

[15] Danilo Erricolo, Algorithm 861: Fortran 90 subroutines for computing the expansion coeffcients of Mathieu functions using Blanch's algorithm, ACM Transactions on Mathematical Software 32 (2006), no. 4, 622-634. MR22290450

[16] M. J. Frisch, Remark on algorithm 352 [S22] Characteristic values and associated solutions of Mathieu's differential equation, Commun. ACM 15 (1972), no. 12, 1074.

[17] S. Goldstein, Mathieu Functions, Camb. Phil. Soc. Trans. 23 (1927), 303-336.

[18] T. M. Habashy, J. A. Kong, and W. C. Chew, Resonance and radiation of the elliptic disk microstrip structure, part I: Formulation, IEEE Trans. Antennas Propagat. 35 (1987), no. 8, $877-886$

[19] T. B. Hansen and A. D. Yaghjian, Low-frequency scattering from two-dimensional perfect conductors, IEEE Trans. Antennas Propagat. 40 (1992), no. 11, 1389-1402.

[20] D. B. Hodge, The calculation of the eigenvalues and eigenvectors of Mathieu's equation, Nasa contractor report, The Ohio State University, Columbus, Ohio, 1972.

[21] E. L. Ince, Tables of elliptic cylinder functions, Roy. Soc. Edin. Proc. 52 (1932), 355-423.

[22] G. C. Kokkorakis and J. A. Roumeliotis, Power series expansions for Mathieu functions with small arguments, Math. Comput. 70 (2001), no. 235, 1221-1235. MR1709153 (2001j:33018)

[23] T. M. Larsen, P. L. E. Uslenghi, and D. Erricolo, Low-frequency behavior of a slotted semielliptical channel, Digest of National Radio Science Meeting (Boulder, CO, USA), Jan 2004, p. 66 .

[24] M. M. S. Lira, H. M. de Oliveira, and R. Jd. S. Cintra, Elliptic-cylindrical wavelets: The Mathieu wavelets, IEEE Signal Process. Lett. 11 (2004), no. 1, 52-55.

[25] É. L. Mathieu, Mémoire sur le mouvement vibratoire d'une membrane de forme elliptique, Jour. de Math. Pures et Appliquées (Jour. de Liouville) 13 (1868), no. 137.

[26] N. W. McLachlan, Theory and Application of Mathieu Functions, Dover Publications, New York, 1964. MR0174808 (30:5001)

[27] J. Meixner and F. W. Schäfke, Mathieusche funktionen und sphäroidfunktionen, Springer, Berlin, 1954. MR0066500 (16:586g)

[28] P. M. Morse and H. Feshbach, Methods of theoretical physics, McGraw-Hill Book Company, Inc., 1953. MR0059774(15:583h)

[29] S. R. Rengarajan and J.E. Lewis, Mathieu Functions of Integraf Orders and Real Arguments, IEEE Trans. Microw. Theory Tech. 28 (1980), no. 3, 276-277.

[30] J. A. Roumeliotis and S.P. Savaidis, Scattering by an infinite circular dielectric cylinder coating eccentrically an elliptic metallic one, IEEE Trans. Antennas Propagat. 44 (1996), no. $5,757-763$.

[31] _ Scattering by an infinite circular dielectric cylinder coating eccentrically an elliptic dielectric cylinder, IEEE Trans. Antennas Propagat. 52 (2004), no. 5, 1180-1185. MR2075887

[32] S. P. Savaidis and J. A. Roumeliotis, Scattering by an infinite elliptic dielectric cylinder coating eccentrically a circular metallic or dielectric cylinder, IEEE Trans. Microw. Theory Tech. 45 (1997), no. 10, 1792-1800.

[33] J. K. Shaw, W. M. Henry, and W. R. Winfrey, Weakly guiding analysis of elliptical core step index waveguides based on the characteristic numbers of Mathieu's equation, J. Lightwave Technol. 13 (1995), no. 12, 2359-2371.

[34] R. B. Shirts, Algorithm 721 MTIEU1 and MTIEU2: Two subroutines to Compute Eignevalues and solutions to Mathieu's differential equation for noninteger order, ACM Trans. Math. Softw. 19 (1993), no. 3, 391-406.

[35] _ - The computation of Eigenvalues and Solutions of Mathieu's differential equation for noninteger order, ACM Trans. Math. Softw. 19 (1993), no. 3, 377-390.

[36] Staff of the computation Laboratory, Tables relating to Mathieu Functions, second edition ed., Applied Mathematics Series, U.S. Government Printing Office, Washington, D.C., 1967, Aug. 1967.

[37] J. A. Stratton, Electromagnetic theory, McGraw-Hill, New York, 1941.

[38] P. L. E. Uslenghi, Exact penetration, radiation and scattering for a slotted semielliptical channel filled with isorefractive material, IEEE Trans. Antennas Propagat. 52 (2004), no. 6, 1473-1480. 
[39] M. Valentino and D. Erricolo, Exact two-dimensional scattering from a slot in a ground plane backed by a semielliptical cavity and covered with an isorefractive diaphram, Radio Sci. 42, no. 6, article number RS6S12, 2007 (DOI 10.1029/2006RS003547).

[40] Visual Numerics, Inc., IMSL Fortran subroutines for mathematical applications, 1994.

[41] E. T. Whittaker and G. N. Watson, A course of modern analysis, 4th ed., Cambridge Univ. Press, 1996, reprinted. MR1424469 (97k:01072)

[42] S. Wolfram, The Mathematica Book, Fifth ed., Wolfram Media, Inc., 2003.

[43] S. Zhang and J.-M. Jin, Computation of special functions, Wiley, New York, 1996. MR:1406797 (97m:65001)

Department of Electrical and Computer Engineering, University of Illinois at Chicago, Chicago, Illinois 60607

E-mail address: tlarse1@comcast.net

Department of Electrical and Computer Engineering, University of Illinois at Chicago, Chicago, Illinois 60607

E-mail address: erricolo@ece.uic.edu

Department of Electrical and Computer Engineering, University of Illinois at Chicago, Chicago, Illinois 60607

E-mail address: uslenghi@uic.edu 